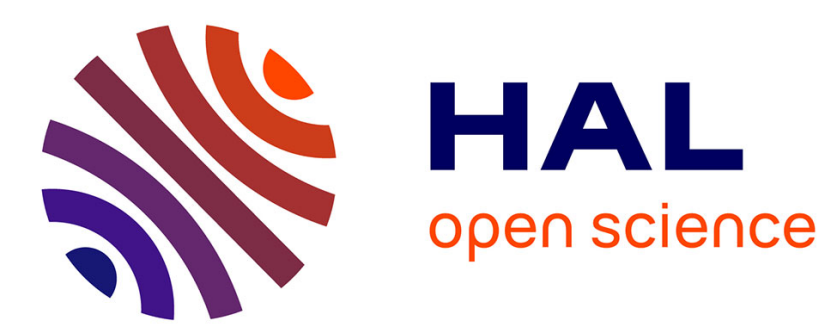

\title{
Design and Simulation-Based Testing of a Prediction Market System Using SIPS for Demand Forecasting
}

Hajime Mizuyama

\section{To cite this version:}

Hajime Mizuyama. Design and Simulation-Based Testing of a Prediction Market System Using SIPS for Demand Forecasting. 19th Advances in Production Management Systems (APMS), Sep 2012, Rhodes, Greece. pp.510-517, 10.1007/978-3-642-40352-1_64 . hal-01472285

\section{HAL Id: hal-01472285 \\ https://hal.inria.fr/hal-01472285}

Submitted on 20 Feb 2017

HAL is a multi-disciplinary open access archive for the deposit and dissemination of scientific research documents, whether they are published or not. The documents may come from teaching and research institutions in France or abroad, or from public or private research centers.
L'archive ouverte pluridisciplinaire HAL, est destinée au dépôt et à la diffusion de documents scientifiques de niveau recherche, publiés ou non, émanant des établissements d'enseignement et de recherche français ou étrangers, des laboratoires publics ou privés. 


\title{
Design and Simulation-Based Testing of a Prediction Market System Using SIPS for Demand Forecasting
}

\author{
Hajime Mizuyama ${ }^{1}$ \\ ${ }^{1}$ Dept. of Industrial and Systems Engineering, Aoyama Gakuin University, \\ Sagamihara Kanagawa 252-5258, Japan \\ mizuyama@ise.aoyama.ac.jp
}

\begin{abstract}
Self-adjustable interval prediction securities (SIPS) are newly proposed prediction securities that are suitable for market-based demand forecasting. The whole feasible region of the demand quantity to be estimated is divided into a fixed number of mutually exclusive and collectively exhaustive prediction intervals. Subsequently, a set of winner-take-all-type securities are issued that correspond to these intervals. Each portion of the securities wins a unitary payoff only if the actual sales volume falls in the corresponding interval. The contracts are called SIPS because the borders between the intervals are dynamically and adaptively self-adjusted to maintain the informativeness of the output forecast distribution. This paper first designs a prediction market system using SIPS equipped with a central market maker and then confirms how the system operates through agent-based simulation.
\end{abstract}

Keywords: Agent simulation, collective intelligence, Delphi method, demand forecasting, prediction markets

\section{Introduction}

In addition to the historical data that are formally owned by a company, fragmentary, dispersed and informal knowledge owned by the company's employees or customers has begun to be treated as a valuable information source for forecasting demand in today's rapidly changing market [1]. Recently, it has been demonstrated that a prediction market can aggregate this dispersed knowledge in a similar, but more efficient manner than the Delphi method [2][3][4]. When a company uses a prediction market to conduct demand forecasting, it usually recruits employees or customers as participants and allows them to trade the fixed-interval prediction securities (FIPS) concerning the demand quantity to be estimated. FIPS are a set of winner-takes-alltype contracts, each of which is tied to a future event that the actual sales volume falls in a specified one among the predetermined set of prediction intervals. Because the intervals are mutually exclusive and collectively comprise the feasible region of the quantity to be estimated, the market prices of the contracts provide a subjective probability distribution of the demand quantity. Some researchers have argued that 
the scope between the most pessimistic and optimistic prior estimates should be divided into around eight equal-width intervals, and they should be used as the prediction intervals together with the regions lower than and higher than the scope [5].

The approach described above has been shown to be effective when tested in an existing company [6]. Despite its utility, however, this approach has several limitations. Most notably, it does not clearly specify the possible scope of the demand quantity a priori. Ironically, as the potential for capturing information from the prediction market grows, so too does the difficulty associated with properly setting the scope. The output forecast distribution depends on a predefined scope and if it is not appropriately set, the entire market session can be rendered meaningless. To resolve this limitation, Mizuyama and Maeda [1] introduced a new type of prediction securities called the self-adjustable interval prediction securities (SIPS). Like FIPS, SIPS are also a set of winner-take-all-type contracts assigned to prediction intervals. Unlike FIPS, however, in SIPS the borders between the intervals dynamically and adaptively self-adjust over the entire feasible region to maintain the informative quality of the output forecast distribution.

This paper first designs a prediction market system using SIPS suitable for demand forecasting, then verifies how the system operates through agent-based simulation. The remainder of the paper will be organized as follows. First, SIPS and the prediction market system using them will be described. Next, the agent-based simulation model for testing the system will be presented. Following this, simulation experiments and their results will be given. Finally, conclusions drawn from these activities will be offered.

\section{Prediction Market System Using SIPS}

\subsection{Self-Adjustable Interval Prediction Security}

Suppose that ten prediction intervals, $I_{1}=\left(-\infty, x_{1}\right], I_{2}=\left(x_{1}, x_{2}\right], \ldots, I_{10}=\left(x_{9}, \infty\right)$, of demand quantity $x$ to be estimated are defined and their corresponding prediction securities are issued. Each unit of these securities is a contract that will pay off a unit amount of money if and only if the realized value of $x$ is actually contained in the corresponding interval. In this situation, the most pessimistic and optimistic prior estimates are $x_{1}$ and $x_{9}$ respectively, and the initial possible scope is $\left(x_{1}, x_{9}\right]$. At the beginning, intervals $I_{2}, I_{3}, \ldots, I_{9}$ have equal width $\left(w_{0}\right)$. If the contracts were FIPS, the borders between intervals $x_{1}, x_{2}, \ldots, x_{9}$ would not be modified until the entire market session is finished.

In case of SIPS, however, the entire market session is divided into several rounds and the divide-merge operation described below is applied at the end of each round so the borders between the intervals can be appropriately updated.

Step 1: Find the interval $I_{n}$ having the highest value of $D_{n}$, which is the evaluation measure to be defined later. If the value of $D_{n}$ is greater than a predetermined threshold, go to Step 2. Otherwise, repeat this step after the next round. 
Step 2: Divide the chosen interval $I_{n}$ :

$$
\begin{aligned}
& x_{k+1}=x_{k} \quad(k=9,8, \ldots, n) \\
& x_{n}=\left\{\begin{array}{cc}
x_{n}-w_{0} & (n=1) \\
\left(x_{n-1}+x_{n}\right) / 2 & (n=2,3, \ldots, 9) \\
x_{n-1}+w_{0} & (n=10)
\end{array}\right.
\end{aligned}
$$

Step 3: Merge the pair of consecutive intervals $I_{m}$ and $I_{m+1}$ having the least countereffect on the evaluation measure:

$$
x_{k}=x_{k+1} \quad(k=m, m+1, \ldots, 10)
$$

Each unit of the prediction security owned by a participant assigned to an interval divided at Step 2 is automatically exchanged for the pair of the securities corresponding to the sub-intervals that resulted from the split. Similarly, a pair of the securities tied to the intervals merged at Step 3 is altered into a unit of the new security corresponding to the merged interval. When a participant holds an uneven number of securities to be merged, some redundant units of either one of them will remain. When this occurs, the redundant units are also exchanged for the new merged security so the prices of the other securities and the proportion of the market value of the participant's assets will not change.

The evaluation measure, $D_{n}$, is defined as follows: The objective of the dividemerge operation is to readjust the definition of prediction intervals so that the hidden collective forecast $f(x)$ can be accurately captured by the price density function $g(x)$, which is defined as:

$$
g(x)=g_{n}=p_{n} / w_{n} \quad\left(x \in I_{n}\right)
$$

where $p_{n}$ is the unitary price of the $n$th security and $w_{n}$ is the width of the $n$th interval (the preset finite value $w_{0}$ is used for $w_{1}$ and $w_{10}$, instead of $\infty$, for convenience). Thus, the effect of the operation can be measured by the extent to which distance between $f(x)$ and $g(x)$ is reduced by the operation. When measuring this effect for each interval, since $f(x)$ is an unknown function, a piecewise quadratic approximation constructed from $g(x)$ is used instead, as shown in Fig. 1, where:

$$
\begin{gathered}
\int_{x_{n-1}}^{x_{n}} \tilde{f}(x) \cdot d x=\int_{x_{n-1}}^{x_{n}} g(x) \cdot d x=w_{n} \cdot g_{n}=p_{n} \\
L_{n}=\left\{\begin{array}{cc}
0 & (n=1) \\
\frac{w_{n} \cdot g_{n-1}+w_{n-1} \cdot g_{n}}{w_{n-1}+w_{n}} & (1<n<10) \\
2 g_{n} & (n=10)
\end{array}\right.
\end{gathered}
$$




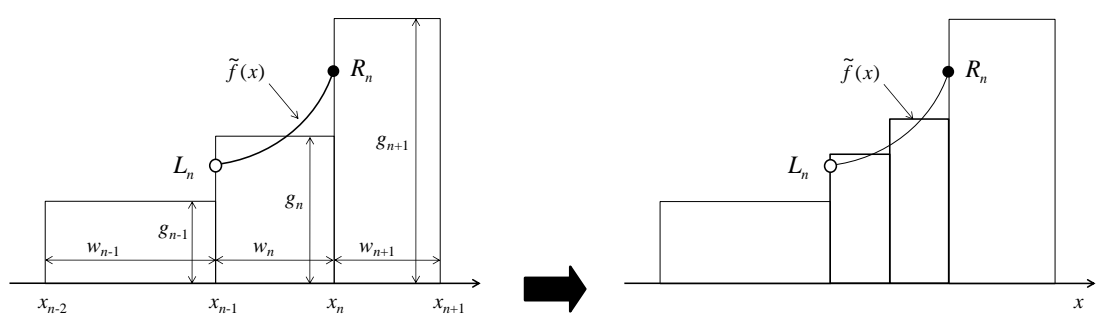

Fig. 1. Piecewise quadratic approximation of $f(x)$ before and after division.

$$
R_{n}=\left\{\begin{array}{cc}
2 g_{n} & (n=1) \\
\frac{w_{n+1} \cdot g_{n}+w_{n} \cdot g_{n+1}}{w_{n}+w_{n+1}} & (1<n<10) \\
0 & (n=10)
\end{array}\right.
$$

According to this approximation, it is possible to estimate how the division of the $n$th interval will change the shape of $g(x)$ as shown in Fig. 1. Thus, the effect of the division can be evaluated by comparing the distance from $g(x)$ to $\tilde{f}(x)$ between the two graphs in Fig. 1. The reduction in distance resulting from the division can be calculated with a formal distance measure between the distributions, such as a Kullback-Leibler distance, L1-norm, L2-norm, and so on. For the sake of simplicity, this paper uses the L1-distance between $g(x)$ before and after the division as a surrogate measure. Thus, the evaluation measure $D_{n}$ of dividing the $n$th interval is defined by:

$$
D_{n}=\left|\int_{x_{n-1}}^{\frac{x_{n-1}+x_{n}}{2}} \tilde{f}(x) \cdot d x-\int_{\frac{x_{n-1}+x_{n}}{2}}^{x_{n}} \tilde{f}(x) \cdot d x\right|=\left|\frac{1}{4}\left(R_{n}-L_{n}\right) \cdot w_{n}\right|
$$

The effect of merging a pair of consecutive intervals can be similarly evaluated.

\subsection{Central Market Maker for SIPS}

Due to the occasional activation of the divide-merge operation, trading SIPS through simple continuous double auction among the participants will be confusing. Thus, this paper provides a computerized market system equipped with a central market maker for trading SIPS. A central market maker in a prediction market accepts any bid/ask requests from a participant as far as she/he agrees with the price offered by the market maker. Accordingly, it resolves the liquidity problem even in a thin market setting.

One of the most well-known and widely-used market-making algorithms for a prediction market is the Logarithmic Market Scoring Rule (LMSR) proposed by Hanson [7][8]. This market maker can handle FIPS. To illustrate, suppose that there are $K$ participants, and participant $k$ has $q_{k n}$ units of the security assigned to the $n$th interval. Given this, the entire security that has been sold thus far is provided by: 


$$
Q_{n}=\sum_{k=1}^{K} q_{k n}
$$

The LMSR defines a cost function based on this variable:

$$
C(\mathbf{Q})=b \cdot \log \left[\sum_{n=1}^{10} \exp \left(Q_{n} / b\right)\right]
$$

where $\boldsymbol{Q}=\left(Q_{1}, Q_{2}, \ldots, Q_{10}\right)$, and determines how much to charge when a participant buys $\Delta \mathbf{q}$ units of the securities using the cost function:

$$
\text { Cost }=C(\mathbf{Q}+\Delta \mathbf{q})-C(\mathbf{Q})
$$

Thus, the unitary price of the $n$th security is determined by:

$$
p_{n}=\frac{d C(\mathbf{Q})}{d Q_{n}}=\frac{\exp \left(Q_{n} / b\right)}{\sum_{i=1}^{10} \exp \left(Q_{i} / b\right)}
$$

Unfortunately, simply applying LMSR to SIPS cannot ensure continuity of the securities' unitary prices in addition to the market value of each participant's assets before and after the divide-merge operation. Thus, to resolve this problem, an explicit prior forecast distribution $f_{0}(x)$ can be introduced and the cost function can be extended accordingly:

$$
C(\mathbf{Q})=b \cdot \log \left[\sum_{n=1}^{10} r_{n} \cdot \exp \left(Q_{n} / b\right)\right]
$$

where:

$$
r_{n}=\int_{x_{n-1}}^{x_{n}} f_{0}(x) \cdot d x
$$

Then, the unitary price of the $n$th security is given by:

$$
p_{n}=\frac{d C(\mathbf{Q})}{d Q_{n}}=\frac{r_{n} \cdot \exp \left(Q_{n} / b\right)}{\sum_{i=1}^{10} r_{n} \cdot \exp \left(Q_{i} / b\right)}
$$

Because of this extension, the division of a certain interval will not affect the respective prices of the securities corresponding to the other intervals and the sum of 
the prices of the divided securities is equivalent to the price of the original security prior to the division. Gao et al. [9] also extended the LMSR to the real line for interval betting. The fundamental concept of their approach was similar to ours, but our approach is specifically tailored for handling SIPS by introducing an explicit prior forecast distribution.

\section{Agent-Based Simulation}

\subsection{Simulation Model}

To test how the proposed prediction market system using SIPS operates, an agent simulation model was developed. In this model, some computerized trading agents traded SIPS with the central market maker introduced above. Each agent had its own subjective forecast distribution and evaluated its risky assets according to the logarithmic utility function and this subjective forecast distribution. For each agent's trading turn, it chose from three options: (1) buying a unit of security corresponding to a certain prediction interval, (2) selling a unit of security corresponding to a certain prediction interval, and (3) buying and selling nothing. Among these, the option that was chosen maximized the posterior subjective expected utility.

\subsection{Simulation Experiments}

Ten trading agents were modeled in the simulation experiments. They were endowed $100 \mathrm{P} \$$ at the beginning of a thirty-round market session ( $\mathrm{P} \$$ is the unit of the play money used in the simulation, and the unitary payoff of the security is $1 \mathrm{P} \$$ ). There were 100 trading turns in each round, and the turns were randomly distributed among the agents. The prior forecast distribution was set as $f_{0}(x)=N(150,100)$; the value of the LMSR parameter $(b)$ was set at 100; and the threshold value of the divide operation was set as 0.03 .

The objective of the simulation experiments was to confirm whether the proposed prediction market system using SIPS can appropriately adjust the scope of forecast according to transaction history. Thus, all the agents were assigned the same Gaussian distribution $f(x)=N(200,20)$ as the subjective forecast distribution, and initial scope of forecast $\left(x_{1}, x_{9}\right]$ which was set as (a) being away from the forecast $(300,400]$, (b) being too wide compared to the forecast $(100,600]$, or (c) being too narrow compared to the forecast $(190,210]$.

The output distributions obtained by FIPS and SIPS are illustrated in Fig. 2, 3 and 4. Simulation results confirmed that in all cases, the scope of the forecast is actually modified step-by-step when SIPS is used. After several rounds, it successfully captured the location of the given subjective forecast distribution. How many rounds it took until the scope has been properly readjusted was contingent upon on the difference between the initial scope and the given distribution. 


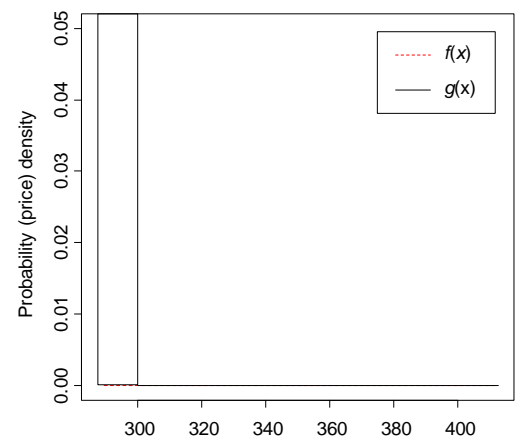

(i) FIPS

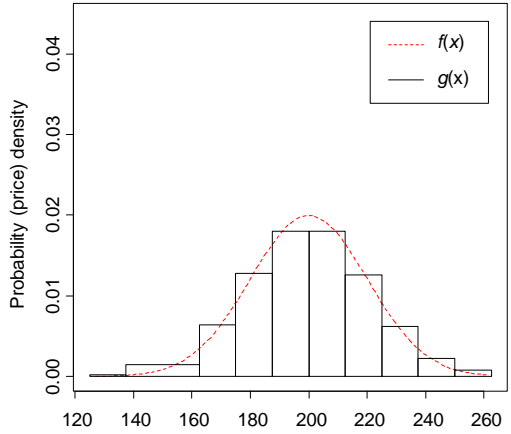

(ii) SIPS

Fig. 2. Output distributions in case (a).

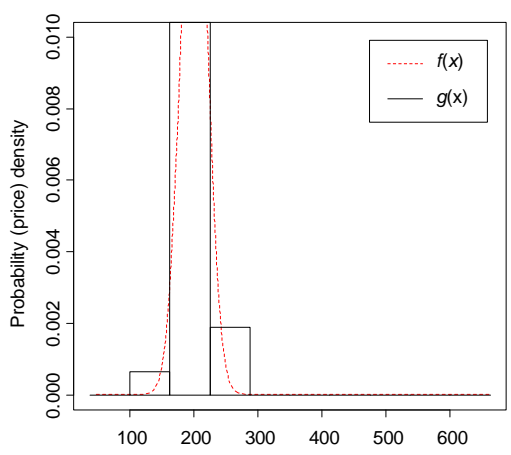

(i) FIPS

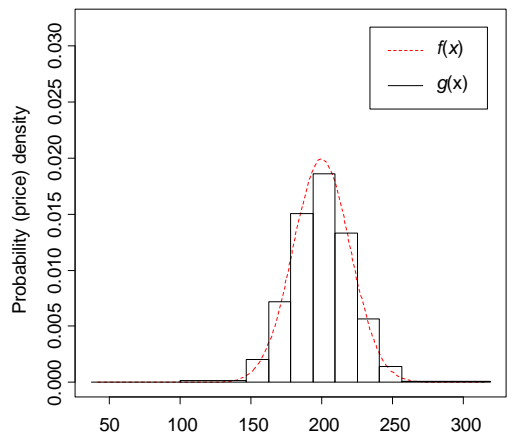

(ii) SIPS

Fig. 3. Output distributions in case (b).
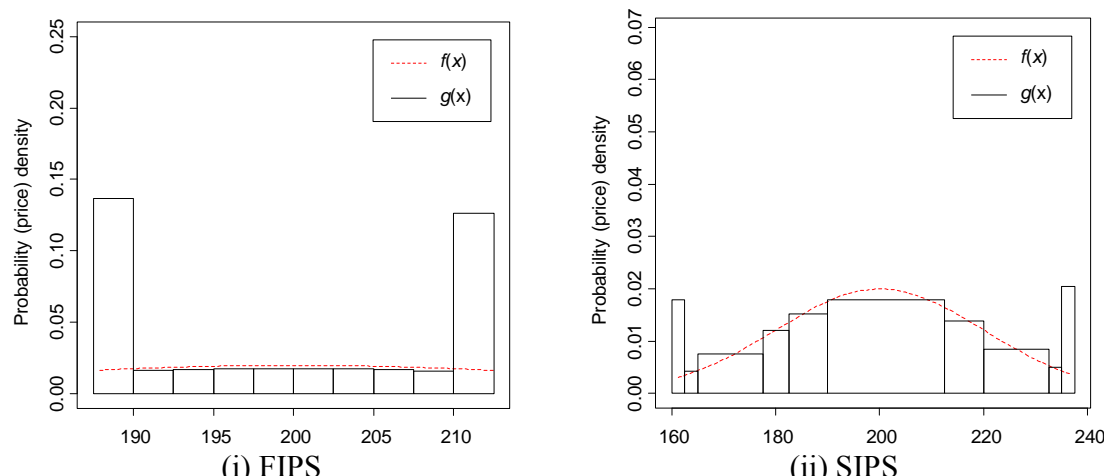

(ii) SIPS

Fig. 4. Output distributions in case (c). 


\section{Conclusions}

This paper details the design of a prediction market system using SIPS equipped with a central market maker and confirms how the system worked through agent-based simulation. As a result, this chapter has demonstrated that the proposed system can properly adjust the scope of a forecast according to transaction history. This paper assumes that the divide-merge operation splits an interval into two equal-length subintervals, but the point at which the interval should be divided can also be treated as a variable that can affect the performance of SIPS. In addition, the number of intervals need not be ten, and changing the number is relatively straightforward.

The system proposed above is now ready to be tested in a real-world setting. Promising areas in which this system could be applied include the estimation of a new product's demand quantity for a given time period after its launch, the estimation of an existing product's demand quantity during a planned sales promotion, and so on. Further, because the performance of the system also depends on the quality of its users' knowledge, it is not suitable for a product on which knowledgeable users are difficult to find. Finally, the proposed system can currently only handle the demand quantity for a single product in a single time period. To utilize the system in a more traditional setting (i.e., one that includes multiple products and periods), it should be extended to handle multiple related demand quantities in parallel. This presents an interesting challenge that should be undertaken in future research in this area.

Acknowledgments. This research was partially supported by the Japan Society for the Promotion of Science, Grant-in-Aid for Scientific Research (B) 20310087.

\section{References}

1. Mizuyama, H., Maeda, Y.: A Prediction Market System Using SIPS and Generalized LMSR for Collective-Knowledge-Based Demand Forecasting, Proceedings of the 40th International Conference on Computers and Industrial Engineering (2010)

2. Plott, C.R.: Markets as Information Gathering Tools. Southern Economic Journal, 67, 1-15 (2000)

3. Pennock, D.M., Lawrence, S., Giles, C.L., Nielsen, F.A.: The Real Power of Artificial Markets. Science, 291, 987-988 (2001)

4. Wolfers, J., Zitzewitz, E.: Prediction Markets. Journal of Economic Perspectives, 18, 107$126(2004)$

5. Ho, T.H., Chen, K.Y.: New Product Blockbusters: The Magic and Science of Prediction Markets. California Management Review, 50, 144-158 (2007)

6. Chen, K.Y., Plott, C.R.: Information Aggregation Mechanisms: Concept, Design and Implementation for a Sales Forecasting Problem. California Institute of Technology, Social Science Working Paper \#1131 (2002)

7. Hanson, R.: Combinatorial Information Market Design. Information Systems Frontiers, 5, 107-119 (2003)

8. Hanson, R.: Logarithmic Market Scoring Rules for Modular Combinatorial Information Aggregation. Journal of Prediction Markets, 1, 3-15 (2007)

9. Gao, X., Chen Y., Pennock, D.M.: Betting on the Real Line. Lecture Notes in Computer Science, 5929, 553-560 (2009) 